Check for updates

Cite this: Phys. Chem. Chem. Phys., 2021, 23, 14363

Received 25th March 2021,

Accepted 8th June 2021

DOI: 10.1039/d1cp01312b

rsc.li/pccp

\title{
From high quality packing to disordered nucleation or phase separation in donor/acceptor interfaces: ClAlPc- $\mathrm{C}_{60}$ on $\mathrm{Au}(111) \dagger$
}

\author{
Esther Barrena, (D) ${ }^{a}$ Rogger Palacios-Rivera, (D) ${ }^{a}$ José I. Martínez (D) ${ }^{b}$ and \\ Carmen Ocal (1D*a
}

\begin{abstract}
The dramatic consequences that the orientation adopted by the molecular dipoles, in diverse arrays of chloroaluminum phthalocyanine (CIAIPC) on $\mathrm{Au}(111)$, have on the ulterior adsorption and growth of $\mathrm{C}_{60}$ are explored by means of an all scanning probe microscopy approach. The unidirectional downwards organization of the molecular dipoles at the first layer reduces charge transfer from the metal to $\mathrm{C}_{60}$. Imbalance between attractive and repulsive interactions of the fullerenes are crucial for their ordered supramolecular aggregation. The effect at the basis of such self-assembling seems to be released by the all upwards dipole orientation adopted on the CIAIPC second layer. The low electronic corrugation of the bilayer results in a higher mobility of the fullerenes which for similar coverages diffuse large distances to reach uncovered first layer regions. Density functional theory calculations corroborate the experimental observations indicating the relevance of charge transfer, potential energy surface corrugation, $\mathrm{C}_{60}$ on-surface diffusion barriers and screening. The structure of the co-adsorbed $\mathrm{C}_{60}$ and CIAIPc layers strongly depends on the deposition sequence. Phase-separation, where each molecule adopts the single-component assembly, occurs if $C_{60}$ is deposited first. The present results contribute to understanding the influence of the dipolar nature of molecular layers on the electronic and structure of donor/acceptor heterojunctions, which is crucial for device design via engineering the energy level alignment at organic-organic and organic-metal interfaces.
\end{abstract}

\section{Introduction}

Understanding the fundamental processes taking place between organic molecules at the donor/acceptor interface, as well as the role of the metal surface, is of enormous importance in optimizing organic electronic devices, such as organic displays, organic field effect transistors, and organic photovoltaic cells. In particular, how competing intermolecular interactions at different scale lengths influence molecular assembly, affecting the interfacial structure, is relevant for practical applications because it controls the band alignment of the molecular systems. Investigating these issues implies, however, the complex task of

\footnotetext{
${ }^{a}$ Institut de Ciència de Materials de Barcelona (ICMAB-CSIC), Campus UAB, Bellaterra, E-08193, Barcelona, Spain. E-mail: cocal@icmab.es

${ }^{b}$ Dept. Nanostructures and Low-dimensional Materials, Institute of Material Science of Madrid (ICMM-CSIC), Campus UAM, C/Sor Juana Inés de la Cruz 3, E-28049 Madrid, Spain

$\dagger$ Electronic supplementary information (ESI) available: Experimental details on the CPD measurements and description of the choice of computed interaction configurtations. Figures showing topography and frequency shift data for the ClAlPc incomplete bilayer and providing the diverse structural models on the surface and gas-phase. See DOI: 10.1039/d1cp01312b
}

considering several weak interactions, such as van der Waals, hydrogen bonds and electrostatic interactions. Moreover, supramolecular organization and self-assembly ${ }^{1,2}$ (the "bottom-up" fabrication method with the lowest energy consumption) on metals and charge transfer between metal surfaces and molecules ${ }^{3,4}$ (essential for charge injection/extraction in molecular electronics) depend on a delicate balance between inter and intramolecular interactions and molecule-surface interactions, which are highly system-dependent. Therefore, the relevance of describing the electronic processes at the molecule/molecule and molecule/metal interfaces, in connection with the structure of the interfaces themselves, is evident for application in devices that involve molecular species in contact with metallic electrodes.

In the workbench of electronic interface design, sandwich structures, where one thin organic layer, acting as spacer, is intercalated between the metallic substrate and the electron acceptor material, have been routinely employed to tune surface work function and control charge transfer from the metal. Self-assembled monolayers covalently bonded to the metal are the most common dielectric spacers used to this end. ${ }^{5-8}$ Richer though much more complex scenarios are open at the donor/ acceptor interface using small organic semiconductor molecules. 
For years, $\mathrm{C}_{60}$ fullerenes have been considered as the archetypal electron acceptor materials due to their large electron affinity and good stability. Because of their practical relevance, a good deal of experimental and theoretical investigations have been devoted to both, electronic properties and structural details, for fullerenes on close-packed metals. ${ }^{9}$ Fewer exist concerning the growth of $\mathrm{C}_{60}$ on top of ordered organic monolayers on metals. ${ }^{10-13}$ In all cases, the subtle balance between moleculemolecule and molecule-substrate interactions is invoked. In particular, the observation of serpentine one dimensional chains or disordered phases have been interpreted in terms of repulsion between fullerene molecules that result charged to some extent after charge transfer from the metal to the fullerene through the intercalated layer. ${ }^{10}$ The possibility of modulating the weight of the contributing interactions by incorporating, under the acceptor molecules, layers made out of molecules with a permanent dipolar moment of diverse orientation emerges as an appealing benchmark. Wei et al. investigated how polymorphism of well-organized monolayers of the polar titanyl phtalocyanine $(\mathrm{TiOPc})^{14}$ affects the interface built upon deposition of $\mathrm{C}_{60}$. Restructuring of the TiOPc monolayer, formation of co-crystalline networks and phase separation into domains with abrupt boundaries were reported. Interestingly, TiOPc molecules initially packed in a tilted (non-planar) configuration were displaced by the $\mathrm{C}_{60}$ deposited on top. ${ }^{15}$

In this context, the system formed by $\mathrm{C}_{60}$ and chloroaluminum phthalocyanine (ClAlPc) is attractive, because both are known to separately yield well-ordered monolayers on $\mathrm{Au}(111)$ which have been well electronically studied. Herein, the effect of the molecular dipoles within a layer intercalated between $\mathrm{C}_{60}$ and the metal is studied by depositing $\mathrm{C}_{60}$ molecules at room temperature (RT) on diverse ordered ClAlPc layers. To understand the influence of dipole orientation, three different scenarios are studied: all Cl-up monolayer, mixed Cl-up and Cl-down monolayer and a bilayer consisting of Cl-up and Cl-down molecules adopting a staggered stacking. Additionally, in an attempt to pursue the role of relative orientation between molecules, the reverse deposition order is also analyzed.

ClAlPc is a nonplanar molecule with permanent electric dipole perpendicular to its molecular $\pi$-plane that can adopt two distinct planar configurations (Cl-up and Cl-down) when adsorbed on metallic surfaces. Competing molecule-molecule and molecule-metal interactions make the adsorption of this dipolar molecule particularly complex. The ClAlPc/Au(111) system has been extensively studied ${ }^{16-19}$ and it is known that, after a moderate post-deposition annealing, the monolayer consists of a highly ordered array of all Cl-up molecules, where the interaction of the conjugated plane and the metal $\left(\pi-\mathrm{d}_{z^{2}}\right.$ coupling) overcomes the dipolar interaction between molecules. Molecules in the second layer adopt the Cl-down configuration and a fourfold adsorption that leads to a staggered stacking, optimizing the electrostatic interaction between first and second layers. It has also been reported that depending on the strain degree of the reconstructed topmost surface of the $\mathrm{Au}(111)$ substrate (that can vary with substrate preparation and postgrowth annealing temperature) the first layer can be built up by the coexistence of Cl-down and Cl-up configurations, as reported for $\mathrm{Au}(111) /$ mica. $^{16}$

The combination of scanning tunneling microscopy (STM) and frequency modulation atomic force microscopy (FM-AFM) with $a b$ initio calculations provide a vast description of the created $\mathrm{C}_{60} / \mathrm{ClAlPc}$ interfaces. The consistency of the energetic and structural landscapes in this particular donor/acceptor system provides some general clues to envisage future fine-tuning of surface work function and design-controlled interfaces.

\section{Methods}

\section{Experimental details}

The Au(111) single crystal (Mateck GmbH, Germany) was prepared by repeated cycles of $\mathrm{Ar}^{+}$sputtering ( $\left.0.8 \mathrm{keV}\right)$ plus annealing at $600{ }^{\circ} \mathrm{C}$ in a preparation chamber equipped with Low Energy Electron Diffraction (LEED) optics and diverse evaporators connected to the STM/AFM characterization chamber. After several cleaning cycles, the observation of a sharp pattern by LEED and the herringbone (HB) reconstruction by STM confirmed a well-ordered clean $\mathrm{Au}(111)$. The HB double rows of discommensuration lines (DL) along the $\langle 112\rangle$ directions were used to establish the crystal azimuth orientation for the structural analysis throughout the present work. This in-situ reference is of relevance since the HB pattern, driven by tensile surface stress, can have a very different local appearance on diverse areas of the same surface. ${ }^{16,20,21}$ Extreme cases are the so called rosetta structure where HB domains converge in a complex star-like structure and the simplest pairwise parallel stripes with consequences on the characteristics of deposited molecular overlayers. $^{20}$ It is worth mentioning that differences in $\mathrm{Au}(111)$ strain release have been shown to affect the orientation of the ClAlPc molecules within the monolayer leading to short-order and disordered mixed (Cl-up and Cl-down) monolayers. ${ }^{16}$ Conversely, the HB patterns have been reported to change upon adsorption of perylene and FePc due to an anisotropic stress induced by charge transfer at the Au interface. ${ }^{22}$

The ClAlPc molecules (Sigma-Aldrich) were purified twice by gradient thermal annealing before being introduced in the ultra-high vacuum (UHV) chamber where they were degassed and sublimated employing a Knudsen cell at $345{ }^{\circ} \mathrm{C}$. Well-ordered layers were obtained in samples annealed for $10 \mathrm{~min}$ at $150{ }^{\circ} \mathrm{C}$ immediately after deposition (post-growth annealing). The $\mathrm{C}_{60}$ molecules were also in situ deposited from the vapour phase using deposition rates of about 2-3 $\AA \min ^{-1}$ using a Knudsen cell $\left(380{ }^{\circ} \mathrm{C}\right)$. The diverse molecular coverages $(\theta)$ at the surface have been estimated from topographic STM images.

The experiments were carried out at RT under a base pressure of $1 \times 10^{-10} \mathrm{mbar}$, using a commercially combined STM/AFM-UHV system Aarhus SPM 150 with KolibriSensor ${ }^{\text {TM }}$ probes $\left(f_{0} \sim 1 \mathrm{MHz}, Q \sim 25000\right)$ and a Nanonis Control System (SPECS Surface Nano Analysis GmbH). The sharp metallic tip was cleaned in situ via $\mathrm{Ar}^{+}$sputtering and, during STM measurements, was maintained oscillating at a constant amplitude $(A=200 \mathrm{pm})$ so that the tip-sample interaction 
was reflected in a frequency shift $(\Delta f)$ from $f_{0}{ }^{23}$ Topographic STM was conducted in the constant current mode and the simultaneous $\Delta f$ was recorded. Typical tunnelling parameters were: sample bias voltages of 1-2 V and currents of 100-200 pA. The in-plane lattice constant, in high resolution images, and step heights, in large-scale images of the in-situ cleaned $\mathrm{Au}(111)$ were employed for calibration of the STM piezo scanner. In the employed setup, the bias voltage is applied to the sample. The local contact potential difference (CPD) of the surfaces was determined from the parabolic dependence of the $\Delta f$ versus bias voltage such that higher CPD corresponds to higher local work function. Details on CPD measurements are provided in the ESI. $\dagger$ All STM/AFM images were analyzed by using the WSxM freeware. ${ }^{24}$

\section{Computational details}

All $a b$ initio calculations, for structural optimization, electronic structure properties and transition-state energy barriers, have been carried out by Density Functional Theory (DFT) as implemented in the plane-wave QUANTUM ESPRESSO simulation package. ${ }^{25}$ One-electron wave functions are expanded in a plane-waves basis with energy cut-offs of 450 and $550 \mathrm{eV}$ for the kinetic energy and the electronic density, respectively. Exchange and Correlation (XC) have been computed in the revised version of the generalized gradient corrected approximation (GGA) of Perdew-Burke-Ernzerhof (rPBE). ${ }^{26}$ Rabe-Rappe-Kaxiras-Joannopoulos (RRKJ) ultrasoft pseudopotentials ${ }^{24-27}$ have been adopted to model the ion-electron interaction. In all the calculations, the Brillouin zone (BZ) has been sampled using optimal $[2 \times 2 \times 1]$ Monkhorst-Pack grids. ${ }^{28}$ The van der Waals (vdW) interaction has been included via an empirical vdW $R^{-6}$ correction (DFT+D3). ${ }^{29}$ Atomic positions have been optimized using a conjugate gradient minimization scheme until the maximum force on any atom was lower than $0.02 \mathrm{eV} \AA^{-1}$. The Fermi level was smeared out using the Methfessel-Paxton approach ${ }^{30}$ with a Gaussian width of $0.01 \mathrm{eV}$, and all energies were extrapolated to $T=0 \mathrm{~K}$. Self-consistency in the electron density to a precision in the total energy better than $10^{-6} \mathrm{eV}$ was achieved. Transition state geometries and energy barriers have been obtained with the Climbing-Image Nudged-Elastic Band method (CI-NEB), ${ }^{31}$ as implemented in QUANTUM ESPRESSO, using 12 intermediate images with a convergence threshold for the norm of the force orthogonal to the reaction path of $0.05 \mathrm{eV} \AA^{-1}$. To estimate the barriers for diffusion from the most stable configurations, we have computed minimum reaction paths (MEP) by the CI-NEB method taking as starting points these equilibrium configurations, stablishing a final state at around $4 \AA$ from the starting point along the diverse directions indicated in each case and including an intermediate step at midway of $2 \AA$ to boost the convergence.

\section{Structural models}

Monolayer and bilayer ClAlPc/Au(111) interfacial models adopted here to theoretically analyze the different $\mathrm{C}_{60}$ adsorption geometries and energies, charge transfers and diffusion transition-state energy barriers, have already been introduced and described in detail in previous literature by our group. ${ }^{19}$ It is important to remark that, in order to eliminate the undesired perpendicular inter-cell interaction after the deposition of the $\mathrm{C}_{60}$ molecules on the different substrates, a minimum distance of $15 \AA$ of vacuum is considered between neighboring cells along the axis perpendicular to the surface in our periodic modelling. A complete description of the substrate models to consider the adsorption of $\mathrm{C}_{60}$ molecules can be found in the ESI. $\dagger$

\section{Results and discussion}

\section{Experimental results}

Well-ordered monolayers of all Cl-up ClAlPc on $\mathrm{Au}(111)$ were prepared using a moderate post-growth annealing as described in the experimental section. The molecules self-arrange in the square lattice $\left(a_{\mathrm{CIAIPc}}=b_{\mathrm{CIAlPc}}=1.38 \pm 0.02 \mathrm{~nm}\right)$ shown in Fig. 1 and full details are given in a previous work. ${ }^{19}$ In this monolayer, all molecules adopt the Cl-up configuration, that is, the layer consists of dipoles with unidirectional downwards orientation $\left(\mu_{\downarrow}\right)$ and their aromatic plane at about $\sim 3 \AA$ from the surface and rotated $10^{\circ}$ with respect to the square unit cell axes.

As it can be seen in wide scan STM images (Fig. 1b and c), large domains of the HB pattern of the reconstructed gold surface are visible underneath and are used to determine the substrate main crystallographic directions. In general, the persistence of the HB is interpreted as an indication of molecular physisorption on a non-strained $\mathrm{Au}(111)$ surface. It is worth mentioning here that the $\mathrm{HB}$ reconstruction is a manifestation of strain relief due to an increased density $(\sim 4 \%)$ of the topmost surface relative to a bulk-truncated (111) surface. Subtle differences in surface relaxation have been observed, for example, in gold films grown on mica, $\mathrm{Au}(111) / \mathrm{mica}^{16}$ Creation of highly mobile adatoms during the preparation procedure may also lead to small density variability and subsequent layer strain fluctuations manifested through HB domain distribution. Since the quality of the organic overlayer depends on it, we will come back to this fact later in this work.

On bare metal (111) surfaces, the $\mathrm{C}_{60}$ molecules are known to form close-packed hexagonal phases. In particular, on $\mathrm{Au}(111)$ it forms diverse well-ordered monolayers with commensurate structures among which the more frequently observed are: $(2 \sqrt{ } 3 \times 2 \sqrt{ } 3) R 30^{\circ}$ and $(7 \times 7) R 0^{\circ},{ }^{32}$ where $R$ is the relative angle

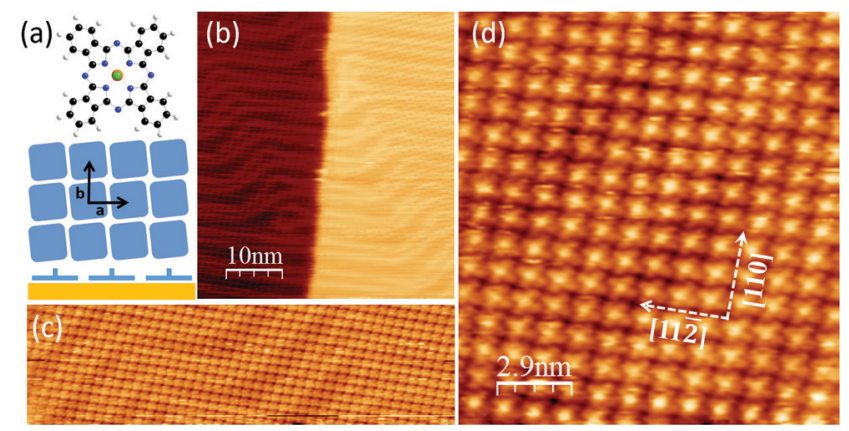

Fig. 1 (a) CIAIPC molecule, top and side view cartoons of the square lattice the $\mathrm{Cl}$-up (downwards dipole) of the molecules within the monolayer. (b-d) STM topographic images for $1 \mathrm{ML}$ of CIAIPC on Au(111); STM parameters: $I=130 \mathrm{pA}$ and $V=+1.25 \mathrm{~V}(\mathrm{~b}$ and $\mathrm{c}$ ) and $V=-1.04 \mathrm{~V}(\mathrm{~d})$. 


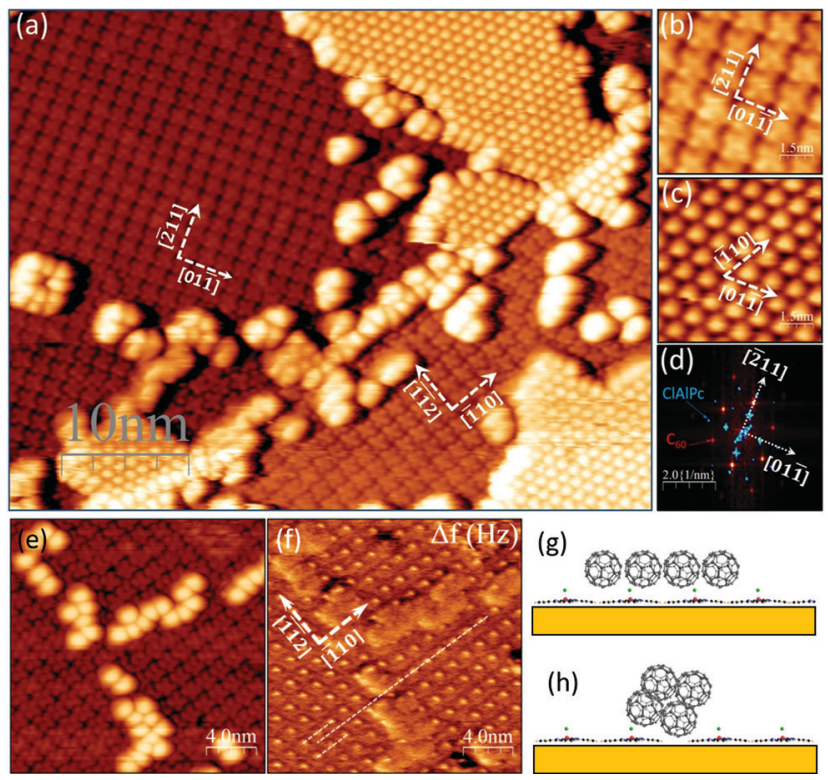

Fig. 2 (a) STM topography ( $50 \mathrm{~nm} \times 39 \mathrm{~nm}$ ) of $\theta \sim 0.3 \mathrm{ML}$ of $\mathrm{C}_{60}$ on $1 \mathrm{ML}$ of CIAIPc on $\mathrm{Au}(111)$ at RT. (b) and (c) are magnified images of the CIAIPC and $\mathrm{C}_{60}$ assemblies on the same terrace; STM parameters: I = $238 \mathrm{pA}$ and $V=+1.97$ V. (d) Superimposed 2D FFT of CIAIPC (blue) and $C_{60}$ (red) domains. Some crystallographic directions are indicated (see text). The cartoons at $(\mathrm{g})$ and $(\mathrm{h})$ illustrate the nucleation of the fullerenes over the monolayer and at defects.

between the lattice orientation of the $\mathrm{C}_{60}$ overlayer and one nearest neighbour (NN) direction of the (111) substrate. Fig. 2 illustrates the result of depositing a submonolayer coverage $(\theta \sim$ $0.3 \mathrm{ML}$ ) of $\mathrm{C}_{60}$ on a complete Cl-up monolayer. The particular surface region shown in Fig. 2a contains two terraces separated by a monoatomic step of $\mathrm{Au}(111)$. As expected from the symmetry relationship between the square adlayer and the hexagonal planes of the fcc crystal, the relative orientation of the square lattices of ClAlPc at each side of the step is $60^{\circ}$. The excellent quality of the $\mathrm{C}_{60}$ close-packed assemblies indicates the good mobility of these molecules at RT on highly ordered regions of the ClAlPc monolayer. The $\mathrm{C}_{60}$ islands have an apparent height of $0.5 \pm$ $0.5 \mathrm{~nm}$ with respect to the underlying ClAlPc monolayer and display a remarkable flatness, with a corrugation of $65 \pm 0.5 \mathrm{pm}$. The molecules exhibit a smooth hemispherical shape. The unit cell of $a_{\mathrm{C} 60}=0.98 \pm 0.05 \mathrm{~nm}$, oriented along the NN directions of the $\mathrm{Au}(111)$. The magnification images of Fig. $2 \mathrm{~b}$ and $\mathrm{c}$ and their corresponding two-dimensional fast Fourier transform (2D-FFT) in Fig. 2d, indicate that the unidirectional epitaxial relationship of

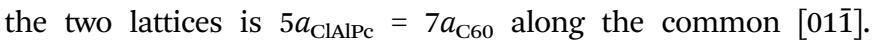
In contrast to the large well-ordered 2D islands, wrinkled chains, isolated molecules and small disordered nuclei of $\mathrm{C}_{60}$ are observed at the lower step edge and at less extended defects of the underlying monolayer on both terraces of Fig. 2a. Such defects are associated either to the presence of disordered regions of ClAlPc molecules close to the steps or to domain boundaries of the square lattice. This is the case shown in Fig. 2e. The existence of equivalent domains laterally displaced half unit cell, with the subsequent frontier along [112], is highlighted by the white dotted lines drawn in the $\Delta f$ image of Fig. $2 \mathrm{f}$.
The similarity between the close-packed hexagonal phase, formed on the ordered ClAlPc monolayer (Fig. 2a and c), and those found for $\mathrm{C}_{60}$ layers on bare metal substrates is remarkable and manifestly contrasts with the quasi-one dimensional organization and low density disordered phases of $\mathrm{C}_{60}$ over zinc phtalocyanine (ZnPc), pentacene or $\alpha$-sexithiophene $(\alpha-6 \mathrm{~T})$ layers on (111) oriented Au and/or Ag surfaces. ${ }^{10,12}$ The lack of $2 \mathrm{D}$ order in those systems has been attributed to the competitive weight of attractive forces and long-range repulsive interactions, regardless of the supramolecular structure of the underlying layers. It is clear that the presence of an intercalated layer between the metal and $\mathrm{C}_{60}$ may alter the $\mathrm{C}_{60}$ intermolecular interactions. For example, in the absence of an interlayer, the repulsive electrostatic interaction between charged molecules, due to charge transfer from the metal to the fullerenes, is effectively screened, therefore allowing molecular selfassembly. Even if charge transfer persists in the presence of an interlayer, the larger distance between the charged $\mathrm{C}_{60}$ and the metal results in diminished screening. For non-charged or weakly charged fullerenes, the hexagonal packing is favoured by strong van der Waals attractions between molecules but is also an indication of their facile diffusion favoured by weak intermolecular interactions with the underlying organic molecules. Indeed, as we will describe below, electronic corrugation influencing diffusion barriers also plays a role and, in the case of an interlayer made out of non-planar molecules with a permanent dipole, the scenario can get much more complicated.

As it is known, the work function $(\phi)$ of the metal's surface is modified by the adsorption of molecules though different processes among which, for example, molecule-substrate charge redistribution and "push-back" effect compete with permanent dipoles in the case of ClAlPc. Because the cases of the single layer (Cl-up) and the bilayer (Cl-down on Cl-up) have already been reported ${ }^{19}$ (see also Fig. S1 in the ESI $\dagger$ ) we only briefly discuss here the consequences of the ClAlPc interlayer on the fullerene layer. Compared to the spatially averaged values obtained by UPS, CPD values obtained by local probes allow spatially resolved measurements on coexisting differentiated regions. Therefore, to get a picture of the energy level alignment, we performed systematic local CPDs on the similarly ordered layers of $\mathrm{C}_{60}$ on the bare and the ClAlPc monolayer. The result is presented in Fig. 3, where the clean Au(111) work function $(\phi=$ $5.35 \mathrm{eV}$ ) as measured separately by UPS is shown as a reference. On top of the well-ordered $\mathrm{C}_{60}$ islands directly grown on gold (see for example Fig. 5a), the work function is reduced by $\Delta \phi=$ $-100 \mathrm{meV}$. This reduction is lower than that reported by UPS for one monolayer. ${ }^{33}$ Otherwise, the effect of depositing an ordered ClAlPc monolayer on $\mathrm{Au}(111)$ is increasing the work function by $\Delta \phi=+70 \mathrm{meV}$, a value that falls by $\Delta \phi=-50 \mathrm{meV}$ when $\mathrm{C}_{60}$ islands are grown on top. In summary, the intercalation of an ordered Cl-up CIAIPc monolayer between $\mathrm{C}_{60}$ and the metal surface slightly raises the work function above that of the clean $\mathrm{Au}(111)$. As we will discuss later, a direct consequence of this vacuum level offset is that electron transfer from the metal surface to $\mathrm{C}_{60}$ becomes unfavourable. Though in this particular system the absolute value of the changes are small, the results 


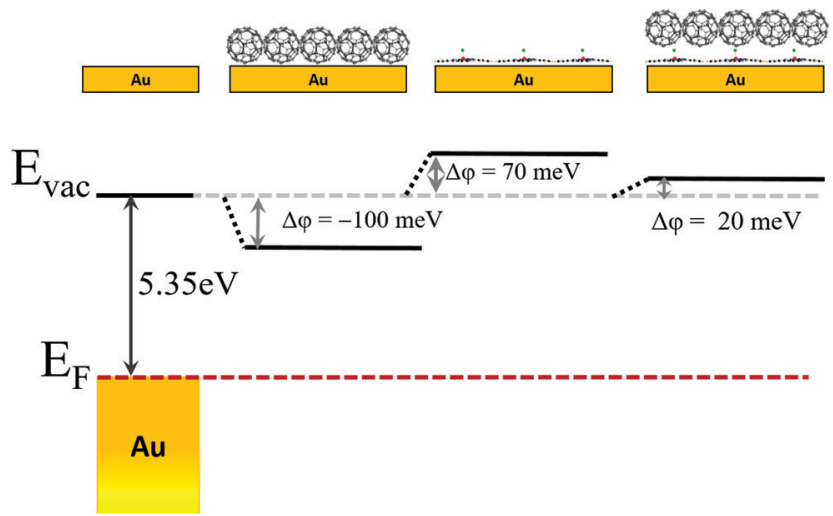

Fig. 3 Vacuum energy levels obtained from CPD measurements on the $\mathrm{C}_{60}$ islands, CIAIPc monolayer and $\mathrm{C}_{60} /$ CIAIPC on $\mathrm{Au}(111)$. The work function of the substrate is used as a reference. Cartoons at the top illustrate the different cases.

open new avenues for interface modification via surface engineering using dipolar molecular layers.

Despite the neat results described above for the all Cl-up monolayer formed over the highly ordered HB reconstruction of the relaxed $\mathrm{Au}(111)$ substrate, the situation becomes more complex if Cl-up and Cl-down configurations coexist on the very same molecular layer. In fact, in the absence of substrate influence, the dipolar interaction between ClAlPc neighbour molecules would favour assemblies with antiparallel orientation of the dipoles (Cl-up and Cl-down). Indeed, disordered or shortrange order mixed 2D layers of ClAlPc have been reported for $\mathrm{Au}(111) / \mathrm{mica}$ and ascribed to differences in the gold surface relaxation. ${ }^{16}$ Such a ClAlPc layer can be seen in Fig. $4 \mathrm{a}$ and $\mathrm{b}$, for a coverage slightly over the monolayer, with the concurrence of the first layer and some individual and small aggregates of a second layer. Close inspection of the lower level reveals that, in addition to the rounded protuberances (bright in both topography and $\Delta \mathrm{f}$ ) typical of molecules in the Cl-up configuration, molecules with Cl-down are also seen (flat four lobes appearance in topography and featureless aspect in $\Delta f$ ). The distribution of each dipole orientation is estimated to be $\sim 50 \%$ in the first layer. In the topographic image shown in Fig. $4 \mathrm{c}$ a bicolour scale has been used to clearly differentiate the deposited $\mathrm{C}_{60}$ (red) from the ClAlPc molecules (green). The contrast between the disordered $\mathrm{C}_{60}$ aggregates on the mixed layer monolayer and the well-packed hexagonal assemblies on the all Cl-up monolayer (Fig. 2a) is obvious. The lack of ordered packing of the fullerenes on the mixed surface is evidenced in the large-scale image of Fig. 4d. Molecules accumulate at extended defects and at small single layer patches, but no order takes place. Then, we can conclude that though the mobility of the molecules is considerably high so they meet and nucleate together, the repulsive interactions seem enough to impede optimal close packing.

After knowing that the misaligned disposition of the permanent dipoles in the first layer (top Fig. 5a) hinders organization of the fullerenes, we explore $\mathrm{C}_{60}$ deposition on an incomplete and defective bilayer. The staggered configuration of dipoles with opposite orientation within first and second layers is shown in

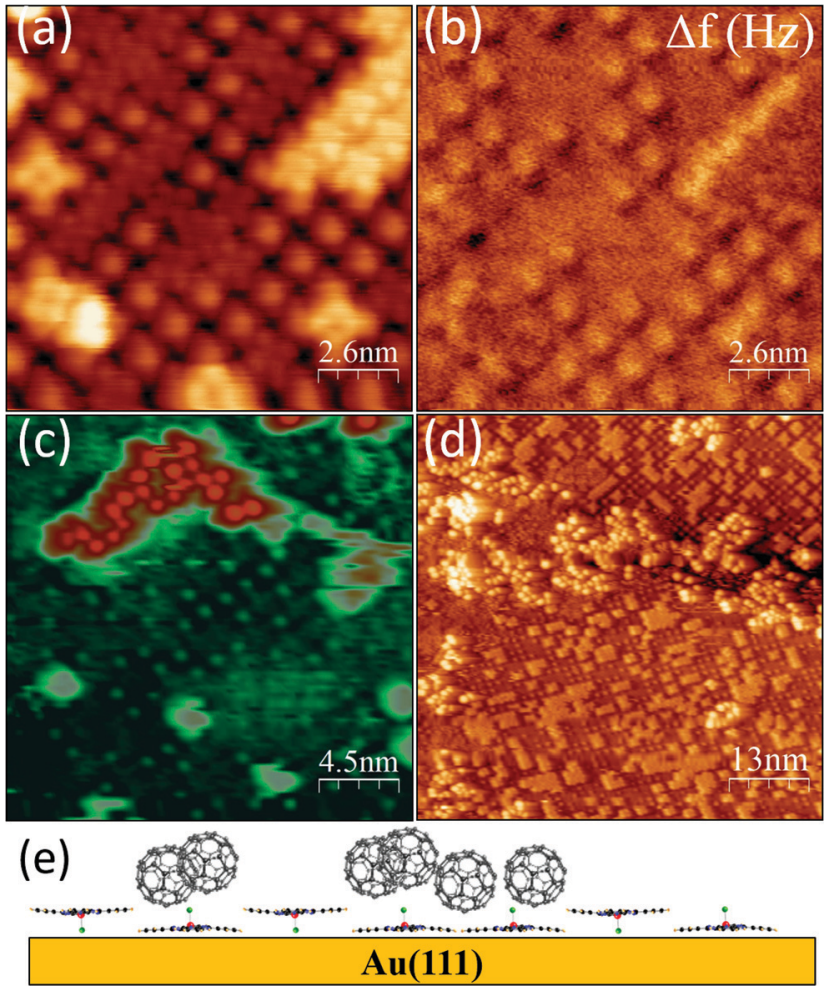

Fig. 4 (a) STM topography and (b) simultaneously measured $\Delta f$ for a mixed (Cl-up and $\mathrm{Cl}$-down) monolayer of ClAIPc. Few second layer molecules are also seen. (c and d) STM topographic images of the same surface after deposition of $\theta \sim 0.1 \mathrm{ML}$ of $\mathrm{C}_{60}$. STM parameters: $I=200 \mathrm{pA}$ and $V=+2.0 \mathrm{~V}$. The bicolor scale of (c) highlights the disordered nucleation of $\mathrm{C}_{60}$ (red).

Fig. 5a. Fig. 5c-f show different areas of the resulting surface after deposition of $\mathrm{C}_{60}(\theta \sim 0.2 \mathrm{ML})$. The most striking observation is that no matter how large the region is, there is neither adsorption nor nucleation on the second ClAlPc layer (Fig. 5c). In fact, and contrary to what happens in the case of the Cl-up monolayer, no nucleation is observed at punctual defects (vacancies) or extended defects (domain boundaries). The molecules diffuse long distances (several tens of unit cells) over this layer to reach empty first layer regions, where due to the Cl-up and Cl-up coexistence, they nucleate in a disordered manner (Fig. $5 \mathrm{c}$ and d) as schematically depicted in the cartoon of Fig. 5e. Remarkably, this process takes place even for relatively large amounts of deposited $\mathrm{C}_{60}$ (Fig. 5f). In the corresponding $\Delta \mathrm{f}$ map of Fig. $5 \mathrm{~g}$, the presence of two different domains and the downwards orientation of the ClalPc molecules forming the second layer are clearly seen. A large number of fullerenes, individually recognized in the images, huddle together at the domain boundary. Outstandingly, at the tallest region of the $\mathrm{C}_{60}$ pile (circled at the $\Delta \mathrm{f}$ image centre) some short-range order is detected, likely indicating the vanishing influence of the layer underneath. The lack of $\mathrm{C}_{60}$ adsorption on the second ClAlPc layer, even for a sufficiently large coverage, is certainly a consequence of a high mobility and large diffusion path of the molecules on the upper level. However, the origin of such low energy barrier for surface diffusion is elusive. As commented above, the key will be found at the electronic corrugation of the ClAlPc layer. 
(a)
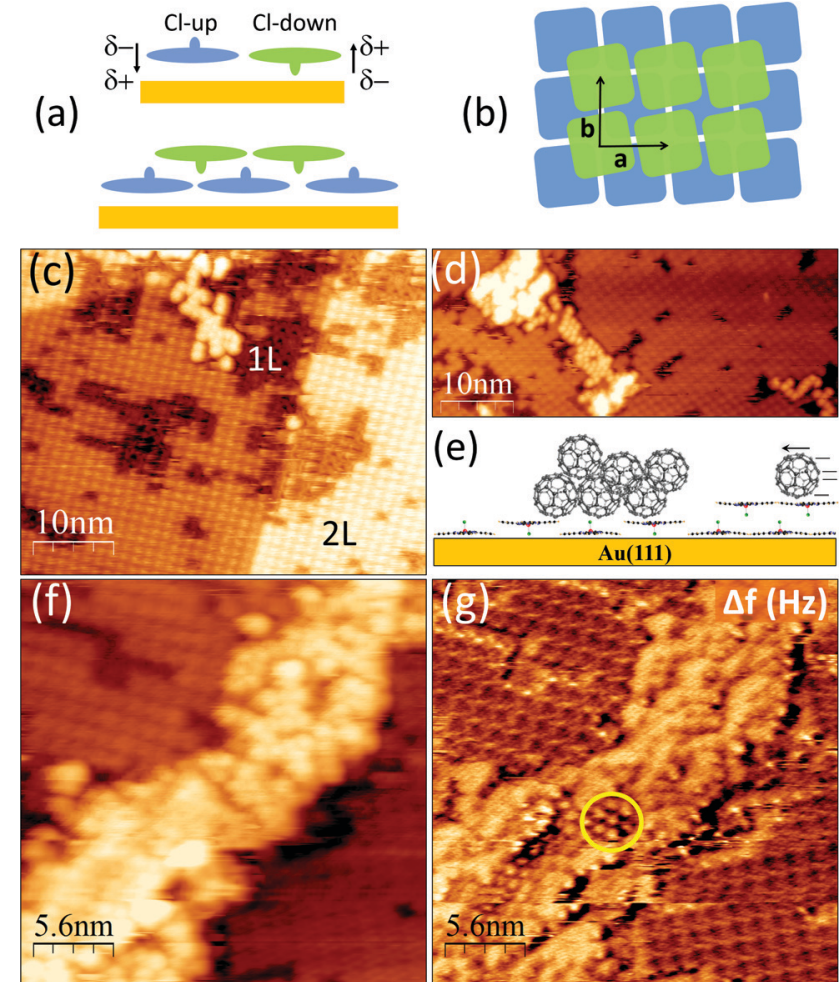

Fig. 5 (a) Cartoons illustrating the side views of a mixed (disordered arrangement of $\mathrm{Cl}$-up and $\mathrm{Cl}$-down) monolayer (top) and an incomplete bilayer (bottom) of CIAIPc. (b) Top view of the CIAIPc bilayer. (c) and (d) STM topography of $\mathrm{C}_{60}$ deposited on a surface consisting of $70 \%$ of a bilayer of CIAIPC and $30 \%$ of a mixed monolayer. (f) STM topography and (g) simultaneously measured $\Delta f$ for a nearly complete bilayer of CIAIPC. STM parameters: (c) $I=357 \mathrm{pA}$ and $V=+2.2 \mathrm{~V}$, (d) $I=200 \mathrm{pA}$ and $V=+2.0 \mathrm{~V}$, and ( $f$ and $\mathrm{g}$ ) $I=150 \mathrm{pA}$ and $V=+1.8 \mathrm{~V}$.

In an attempt to rationalize from a theoretical perspective the different scenarios experimentally observed after the deposition of $\mathrm{C}_{60}$ molecules on: (i) well-ordered monolayers of all Cl-up ClAlPc on $\mathrm{Au}(111)$, and (ii) ClAlPc bilayer on $\mathrm{Au}(111)$, we have carried out a large and time-consuming battery of DFT-based calculations. A detailed structural description of the two types of ClAlPc adlayers on $\mathrm{Au}(111)$ can be found elsewhere. ${ }^{19}$ The factors employed for the choice of the possible configurations, for $\mathrm{C}_{60}$ on both adlayers as well as for the gas-phase interaction geometries, are described in the ESI. $\dagger$ Interestingly, in the gas phase, the calculation indicates that charge transfer and interaction energy strongly depend on the interaction geometry and relative orientation between the $\mathrm{C}_{60}$ and ClAlPc molecules (ESI, $\dagger$ Fig. S5). We have computed equilibrium geometries (keeping fixed the two bottommost $\mathrm{Au}(111)$ layers during the optimization process), adsorption energies and Bader charge transfers from the substrate for a $\mathrm{C}_{60}$ molecule (per unit cell) on a monolayer of Cl-up ClAlPc on $\mathrm{Au}(111)$ on three representative adsorption sites (for each analysing two cases: the interacting lowest part of the $\mathrm{C}_{60}$ being a $\mathrm{C}_{6}$-ring or a $\mathrm{C}_{2}$-bridge). The different adsorption sites and their corresponding optimized geometries obtained from the calculations are shown in Fig. 6. Most stable configurations are those with the $\mathrm{C}_{60}$ molecule lying on the terminal $\mathrm{Cl}$ atom (Fig. 6a and b), with adsorption
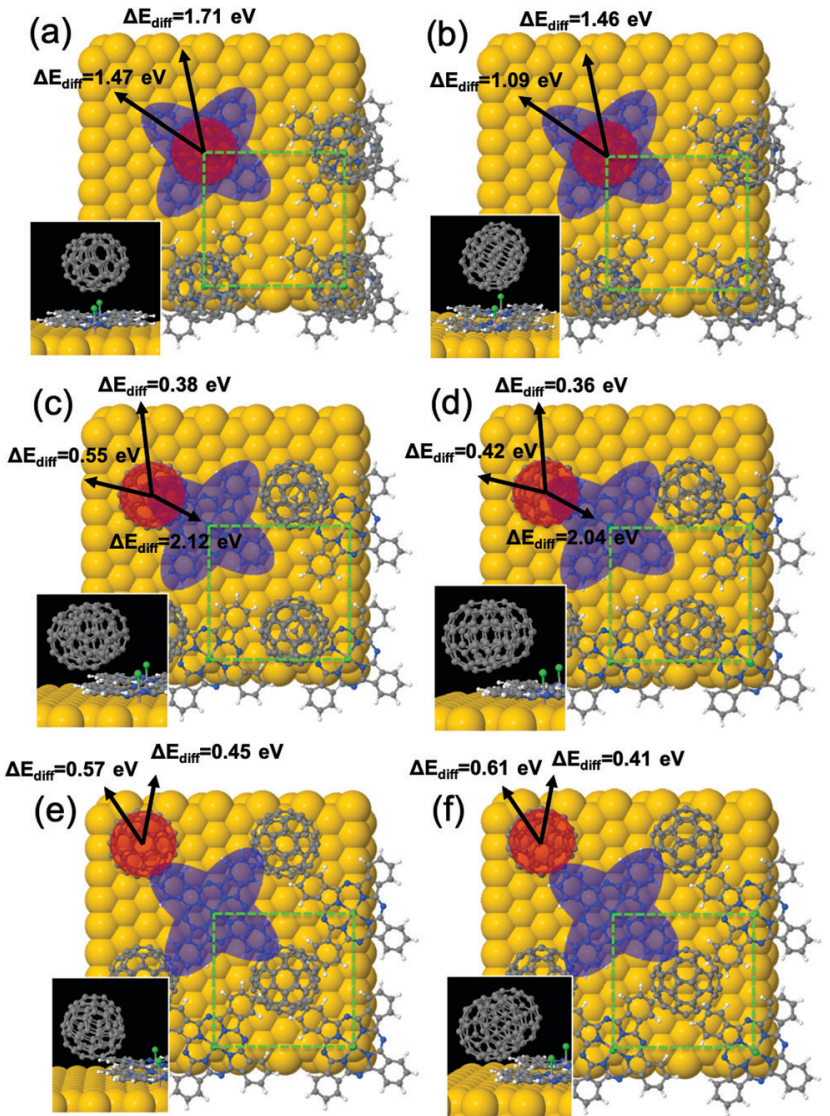

Fig. 6 Top and side (insets) pictorial views of the DFT-optimized structural models for the adsorption of $\mathrm{C}_{60}$ molecules on an ordered monolayer of all $\mathrm{Cl}$-up CIAIPc on Au(111) for six representative adsorption sites (a-f). The unit cell used in the calculations is shown as a light-green dashed-lined box. Blue and red shadowed regions represent the Cl-up CIAIPC and $\mathrm{C}_{60}$ molecules, respectively. Computed $\mathrm{Cl}$-NEB diffusion barriers $\left(\Delta E_{\text {diff; }}\right.$ in $\mathrm{eV}$ ) are indicated along specified directions (black arrows) for each case. More details are given in Fig. S2 of the ESI. $\dagger$

energies per $\mathrm{C}_{60}$ of 1.31 and $1.14 \mathrm{eV}$ for the interaction with a bottommost $\mathrm{C}_{6}$-ring or a $\mathrm{C}_{2}$-bridge, respectively. These values can be directly compared with 1.12 and $0.81 \mathrm{eV}$ obtained for the gasphase interaction between $\mathrm{C}_{60}$ and ClAlPc in the same interaction configurations (Fig. S5 in the ESI $\dagger$ ). In the other on-surface configurations analysed (Fig. 6c-f) the $\mathrm{C}_{60}$ molecule lies on the molecular "wings" with residual lateral interactions with the $\mathrm{Cl}$ atoms, yielding adsorption energies ranging between 0.6 and $0.75 \mathrm{eV}$ governed by vdW interactions, nonetheless sufficient to anchor the molecules to the surface.

We have also computed the charge transfer processes (see the Methods section) occurring after the formation of the interface of $\mathrm{C}_{60}$ on the Cl-up ClAlPc/Au(111). We obtain charge transfers from the substrate to the $\mathrm{C}_{60}$ molecules of 0.22 and $0.16 e^{-}$for the configurations in Fig. 6a and $\mathrm{b}$, respectively. Within the theoretical formalism employed in this work, the charge transfer from the $\mathrm{Au}(111)$ to the $\mathrm{C}_{60}$ in its most stable and fully relaxed $(2 \sqrt{ } 3 \times 2 \sqrt{ } 3) R 30^{\circ}$ periodic geometry ${ }^{34,35}$ is $0.28 e^{-}$. A value significantly lower than the $0.8 e^{-}$estimated by resonant photoemission for the $\mathrm{C}_{60} / \mathrm{Au}(111)$ interface. $^{36}$ 
Although the computed values are of the same order with or without the ClAlPc monolayer, they are higher by $30 \%$ and $75 \%$ for $\mathrm{C}_{60} / \mathrm{Au}(111)$ than for the configurations in Fig. $6 \mathrm{a}$ and $\mathrm{b}$. This can be considered as a non-negligible reduction of the charge transfer to $\mathrm{C}_{60}$ in the presence of the unidirectional dipole interlayer, and therefore diminishing the chargeinduced repulsion between molecules. Besides, upon $\mathrm{C}_{60}$ adsorption the ClAlPc adlayer accumulates, on average per molecule, an excess of electronic charge of $0.19 e^{-}$, appreciably lower than without $\mathrm{C}_{60}$ molecules $\left(0.32 e^{-}\right) .{ }^{19}$ The consequences in molecular assembling are reinforced by the calculation of diffusion barriers from the most stable configurations in Fig. 6 . The calculations yield on-surface diffusion barriers ranging between $0.36 \mathrm{eV}$ and $1.71 \mathrm{eV}$, justifying only relative RT diffusion of the $\mathrm{C}_{60}$ molecules on the Cl-up ClAlPc monolayer. The low repulsion between $\mathrm{C}_{60}$ molecules, their moderate diffusion, summed to the intensity of the interaction with the underlying ClAlPc, favours the stabilization of the $\mathrm{C}_{60}$ molecules and leads to the formation of the robust close-packing of the fullerenes observed in Fig. 2a and c.

In a following step, we have computed equilibrium geometries, adsorption energies and Bader charge transfers from the substrate for a $\mathrm{C}_{60}$ molecule (per unit cell) on the ClAlPc bilayer on $\mathrm{Au}(111)$. Fig. 7 shows the 4 different adsorption sites studied (two with the interacting lowest part of the $\mathrm{C}_{60}$ being a $\mathrm{C}_{2}$-bridge and two being $\mathrm{a}_{6}$-ring), and the corresponding optimized geometries. In this case the most stable configurations are those with the $\mathrm{C}_{60}$ molecule lying on the $\mathrm{Al}$ atom of the Cl-down ClAlPc molecules with a bottommost $\mathrm{C}_{2}$-bridge (Fig. 7a) and a $\mathrm{C}_{6}$-ring (Fig. 7b). The adsorption energies per $\mathrm{C}_{60}$ in each configuration are 0.48 and $0.56 \mathrm{eV}$, respectively, with charge transfers below $0.02 e^{-}$, in a clear physisorption regime. For the other two cases (Fig. $7 \mathrm{c}$ and d), adsorption energy and charge transfer are around $0.4 \mathrm{eV}$ and $<0.01 e^{-}$, respectively. The net charge accumulated in the bilayer of around $0.1 e^{-}$in all cases. The scenario has completely changed with respect to the adsorption on the Cl-up ClAlPc monolayer. Now, the nature of the interaction is merely vdW and adsorption energies are rather low. In order to evaluate the diffusion rate of the $\mathrm{C}_{60}$ molecules, we have computed the diffusion barriers, similarly to the previous case. The result of these calculations shows in all cases diffusion barriers $<0.11 \mathrm{eV}$ independently of the diffusion direction tested, which justifies a high diffusion capability of the molecules deposited on the bilayer. This effect is clearly driven by the low electronic corrugation of the bilayer, leaving the $\mathrm{C}_{60}$ molecules free to diffuse long distances on the surface until reaching highly-reactive on-surface defects or empty first layer regions, as experimentally evidenced.

With a last experimental case study, we will show that the structure of co-adsorbed $\mathrm{C}_{60}$ and ClAlPc layers strongly depends on the deposition sequence. As already known, if $\mathrm{C}_{60}$ is deposited first, islands of compact hexagonal packing are formed on the bare $\mathrm{Au}(111)$. For a coverage of $\theta \sim 0.5 \mathrm{ML}$, the surface is made out of crystalline $\mathrm{C}_{60}$ patches surrounded by bare substrate (Fig. 8a-c). Some defects in the form of individual fullerenes with lower apparent height (darker in the image) is characteristic of $\mathrm{C}_{60}$ on $\mathrm{Au}(111) .{ }^{37}$ The result of (a)
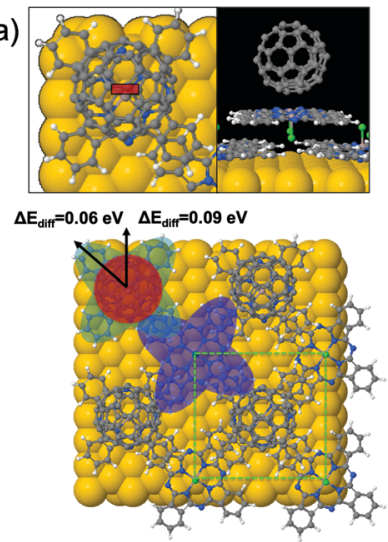

(c)

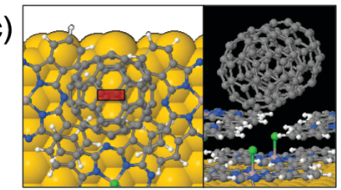
$\Delta E_{\text {diff }}=0.08 \mathrm{eV}$

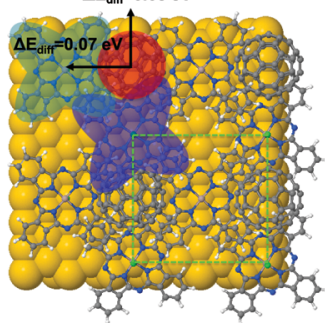

(b)

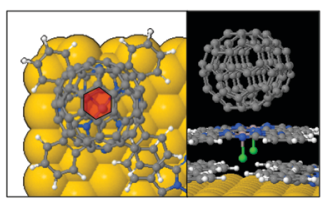

$\Delta E_{\text {diff }}=0.09 \mathrm{eV} \Delta E_{\text {diff }}=0.11 \mathrm{eV}$

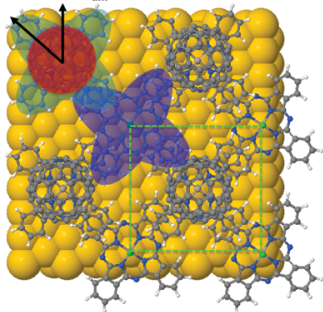

(d)
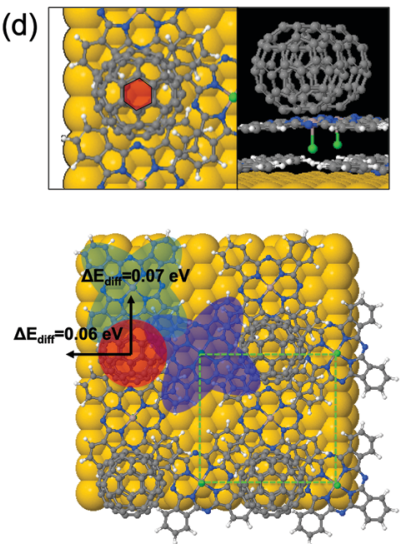

Fig. 7 Top and side views of the DFT-optimized structural models for the adsorption of $\mathrm{C}_{60}$ on an ordered bilayer of $\mathrm{Cl}$-up (bottom layer) and $\mathrm{Cl}$-down (top layer) CIAIPc on Au(111). Four different fully DFT-optimized configurations are depicted resulting from the consideration of two representative adsorption sites and two different $C_{60}$ orientations (interacting via a $\mathrm{C}_{6}$-ring or a $\mathrm{C}_{2}$-bridge). The most stable configurations are those with the $\mathrm{C}_{60}$ molecule lying on the $\mathrm{Al}$ atom of the $\mathrm{Cl}$-down CIAIPC molecules with a bottommost $C_{2}$-bridge (a) and a $C_{6}$-ring (b). Terminal $\mathrm{C}_{2}$-bridges (left) and $\mathrm{C}_{6}$-rings (right) are represented by red rectangles and hexagons, respectively, in the insets. The unit cell used in the calculations is shown as a light-green dashed-lined box. Blue, green and red shadowed regions represent the $\mathrm{Cl}$-up $\mathrm{CIAIPC}, \mathrm{Cl}$-down $\mathrm{CIAIPC}$ and $\mathrm{C}_{60}$ molecules, respectively. Computed $\mathrm{Cl}-\mathrm{NEB}$ diffusion barriers $\left(\Delta E_{\text {diff; }}\right.$ in $\left.\mathrm{eV}\right)$ are indicated along specified directions (black arrows) for each case. More details are given in Fig. S3 and S4 in the ESI. $\dagger$

the subsequent deposition of 1ML of ClAlPc on such a surface is shown in Fig. 8b. Remarkably, instead of covering the entire exposed surface (bare gold and $\mathrm{C}_{60}$ ), the impinging phtalocyanines do not adsorb on the fullerene layer but nucleate at the pristine substrate regions (Fig. 8d). Lateral phase-separation occurs, where each molecule adopts the well-ordered supramolecular order corresponding to the structures of the single-component cases. As the amount of ClAlPc (1ML) is about twice that needed to fill the bare $\mathrm{Au}(111)$ regions, the phtalocyanines pile up with the supramolecular packing of the bilayer (Fig. 5b). ${ }^{19}$ The segregation between molecules, both on the same terrace and on neighbouring terraces, can be seen in Fig. 8e. ClAlPc molecules in the first layer are in close contact with the $\mathrm{C}_{60}$ domains, while because the square lattice of the second layer is shifted a half unit cell in each direction, a thin empty rim exists at the frontier with $\mathrm{C}_{60}$ (see Fig. $8 \mathrm{~b}$ and e). 

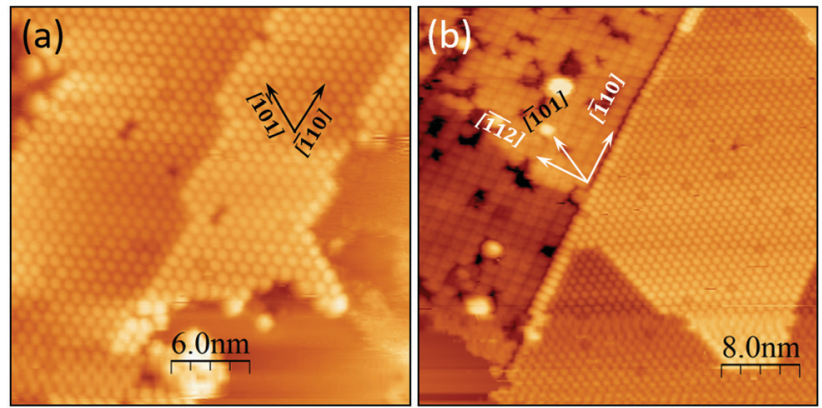

(c)

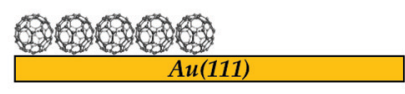

(d)
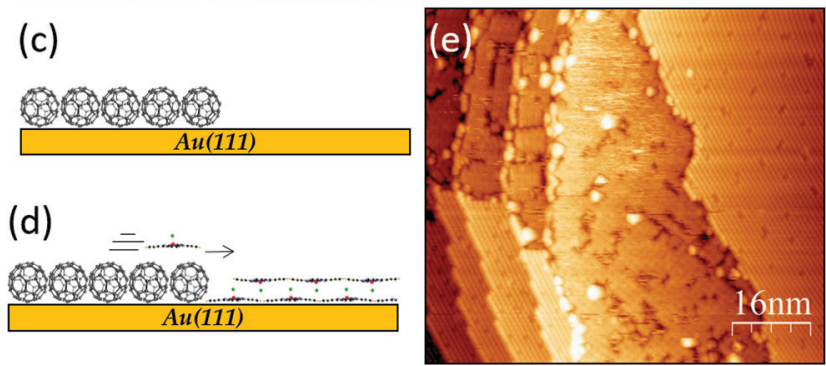

Fig. 8 STM topographic images of (a) $\theta \sim 0.5 \mathrm{ML}$ of $\mathrm{C}_{60}$ on $\mathrm{Au}(111)$ (b) The same surface after $\theta \sim 1 \mathrm{ML}$ of CIAIPC. (c) and (d) are cartoons illustrating the surface in (a) and (b), respectively. CIAIPc does not adsorb but diffuses on $\mathrm{C}_{60}$. (e) Larger area showing phase segregation of CIAIPC and $\mathrm{C}_{60}$. STM parameters: (a) $I=120 \mathrm{pA}$ and $V=+1.8 \mathrm{~V}$, (b) $I=180 \mathrm{pA}$ and $V=+1.5 \mathrm{~V}$, and (e) $I=200 \mathrm{pA}$ and $V=+2.0 \mathrm{~V}$.

Though a very low $\triangle \mathrm{CPD} \sim 50 \mathrm{meV}$ was measured between the two phases (see Fig. 3 and ref. 19) and conversely to abrupt boundaries with negligible dipole reported for phase separated $\mathrm{C}_{60}$-TiOPc films on $\mathrm{Ag}(111){ }^{15,38}$ disconnected boundaries between $\mathrm{C}_{60}$ and ClAlPc domains on $\mathrm{Au}(111)$ may influence the electronic coupling and electrical potential offset across the donor-acceptor interface of relevance for charge separation in OPV devices.

\section{Conclusions}

We provide experimental evidence of the influence of an intercalated layer of dipolar ClAlPc molecules on the charge transfer between $\mathrm{Au}(111)$ and the electron acceptor $\mathrm{C}_{60}$. Both electronic and structural details of the ClAlPc layers become relevant for the organic-organic interface properties. The significance of the charge transfer and energy alignment across the intercalated ClAlPc film has consequences in the balance between attractive and repulsive interactions and, therefore, in the aggregation of the fullerenes. On top of the unidirectional monolayer of ClAlPc, with all molecules in the Cl-up configuration (downwards dipoles), $\mathrm{C}_{60}$ self-assembles in a robust close-packed hexagonal order. Conversely, $\mathrm{C}_{60}$ piles up disorderly when deposited on a monolayer with mixed orientation of the molecular dipoles (disordered coexistence of Cl-up and Cl-down). The overall compensation of dipoles in such mixed configuration would allow competition, between van der Waals intermolecular attraction and repulsion between charged $\mathrm{C}_{60}$ molecules, to manifest by a lack of order in their packing. Remarkably, despite the expected low electrostatic repulsion due to small charge transfer, $\mathrm{C}_{60}$ does not adsorb on the ClAlPc bilayer (topmost layer made out of all
Cl-down, upwards dipoles) but nucleates at uncovered patches of the first layer. We ascribe this experimental evidence at the early stages of $\mathrm{C}_{60}$ growth to a lower adsorption energy and the lower diffusion barriers calculated over the bilayer stack with respect to the monolayer. The structure of co-adsorbed $\mathrm{C}_{60}$ and ClAlPc layers strongly depend on the deposition sequence. If fullerenes are deposited first, phase-separation occurs, where each molecule adopts the structure of the single component cases on clean $\mathrm{Au}(111)$.

\section{Conflicts of interest}

There are no conflicts to declare.

\section{Acknowledgements}

This work has been supported by the Spanish Government under projects PID2019-110907GB-I00, MAT2016-77852-C2-1-R and MAT2017-85089-C2-1-R (AEI/FEDER, UE) and the "Severo Ochoa" Program for Centres of Excellence in R\&D (CEX2019000917-S). We also acknowledge the Generalitat de Catalunya grant 2017 SGR668. J. I. M. acknowledges Comunidad de Madrid via "Programa de Investigación Tecnologías 2018" (FOTOART-CM S2018/NMT-4367), and the EU Innovation Program under grant agreement 881603 (GrapheneCore3Graphene-based disruptive technologies).

\section{References}

1 A. Kühnle, Self-assembly of organic molecules at metal surfaces, Curr. Opin. Colloid Interface Sci., 2009, 14, 157-168.

2 C. A. Palma, M. Cecchini and P. Samori, Predicting selfassembly: From empirism to determinism, Chem. Soc. Rev., 2012, 41, 3713-3730.

3 N. Koch, S. Duhm, J. P. Rabe, A. Vollmer and R. L. Johnson, Optimized hole injection with strong electron acceptors at organic-metal interfaces, Phys. Rev. Lett., 2005, 95, 237601.

4 S. Braun, W. R. Salaneck and M. Fahlman, Energy-level alignment at organic/metal and organic/organic interfaces, Adv. Mater., 2009, 21, 1450-1472.

5 G. Witte and C. Wöll, Molecular beam deposition and characterization of thin organic films on metals for applications in organic electronics, Phys. Status Solidi, 2008, 205, 497-510.

6 B. de Boer, A. Hadipour, M. M. Mandoc, T. van Woundenbergh and P. W. M. Blom, Tuning of metal work functions with self-assembled monolayers, Adv. Mater., 2005, 17, 621-625.

7 J. Ch. Love, L. A. Estroff, J. K. Kriebel, R. G. Nuzzo and G. M. Whitesides, Self-assembled monolayers of thiolates on metals as a form of nanotechnology, Chem. Rev., 2005, 105, 1103-1169.

8 M. Singh, N. Kaur and E. Comini, The role of self-assembled monolayers in electronic devices, J. Mater. Chem. C, 2020, 8, 3938-3955. 
9 X.-Q. Shi, M. A. Van Hove and R.-Q. Zhang, Survey of structural and electronic properties of $\mathrm{C}_{60}$ on close-packed metal surfaces, J. Mater. Sci., 2012, 47, 7341-7355.

10 J. Niederhausen, P. Amsalem, A. Wilke, R. Schlesinger, S. Winkler, A. Vollmer, J. P. Rabe and N. Koch, Doping of $\mathrm{C}_{60}$ (sub)monolayers by Fermi-level pinning induced electron transfer, Phys. Rev. B: Condens. Matter Mater. Phys., 2012, 86, 081411.

11 W. Jin, Q. Liu, D. B. Dougherty, W. G. Cullen, J. E. ReuttRobey, J. Weeks and S. W. Robey, $\mathrm{C}_{60}$ chain phases on ZnPc/ $\mathrm{Ag}(111)$ surfaces: Supramolecular organization driven by competing interactions, J. Chem. Phys., 2015, 142, 101910.

12 L. Chen, W. Chen, H. Huang, H. L. Zhang, J. Yuhara and A. T. S. Wee, Tunable arrays of $\mathrm{C}_{60}$ molecular chains, Adv. Mater., 2008, 20, 484-488.

13 H. L. Zhang, W. Chen, L. Chen, H. Huang, X. S. Wang, J. Yuhara and A. T. S. Wee, $\mathrm{C}_{60}$ molecular chains on a-sexithiophene nanostripes, Small, 2007, 3, 2015-2018.

14 X. Liu, Y. Wei, J. E. Reutt-Robey and S. W. Robey, Dipoledipole interactions in TiOPc adlayers on Ag, J. Phys. Chem. C, 2014, 118(7), 3523-3532.

15 Y. Wei and J. E. Reutt-Robey, Molecular interface formation in titanyl phthalocyanine $-\mathrm{C}_{60}$ monolayer films, J. Phys. Chem. C, 2012, 116, 23773-23778.

16 T. Niu, Surface strain mediated dipole alignment of ClAlPc on Au(111), Appl. Phys. Lett., 2015, 106, 161601.

17 Y. L. Huang, W. Chen, F. Bussolotti, T. C. Niu, A. T. S. Wee, $\mathrm{N}$. Ueno and S. Kera, Impact of molecule-dipole orientation on energy level alignment at the submolecular scale, Phys. Rev. B: Condens. Matter Mater. Phys., 2013, 87, 085205.

18 Y. L. Huang, Y. Lu, T. C. Niu, H. Huang, S. Kera, N. Ueno, A. T. S. Wee and W. Chen, Reversible single-molecule switching in an ordered monolayer molecular dipole array, Small, 2012, 8, 1423-1428.

19 S. Matencio, R. Palacios-Rivera, J. I. Martínez, C. Ocal and E. Barrena, Chiral organization and charge redistribution in chloroaluminum phthalocyanine on $\mathrm{Au}(111)$ beyond the monolayer, J. Phys. Chem. C, 2018, 122, 16033-16041.

20 J. V. Barth, H. Brune, G. Ertl and R. J. Behm, Scanning tunneling microscopy observations on the reconstructed $\mathrm{Au}(111)$ surface: Atomic structure, long-range superstructure, rotational domains, and surface defects, Phys. Rev. B: Condens. Matter Mater. Phys., 1990, 42, 9307-9318.

21 T. J. Roussel, E. Barrena, C. Ocal and J. Faraudo, Predicting supramolecular self-assembly on reconstructed metal surfaces, Nanoscale, 2014, 6, 7991-8001.

22 J. T. Sun, L. Gao, X. B. He, Z. H. Cheng, Z. T. Deng, X. Lin, H. Hu, S. X. Du, F. Liu and H.-J. Gao, Surface reconstruction transition of metals induced by molecular adsorption, Phys. Rev. B: Condens. Matter Mater. Phys., 2011, 83, 115419.

23 T. R. Albrecht, P. Grütter, D. Horne and D. Rugar, Frequency modulation detection using high-Q cantilevers for enhanced force microscope sensitivity, J. Appl. Phys., 1991, 69, 68-673.

24 I. Horcas, R. Fernández, J. M. Gómez-Rodríguez, J. Colchero, J. Gómez-Herrero and A. M. Baro, WSxM: A software for scanning probe microscopy and a tool for nanotechnology, Rev. Sci. Instrum., 2007, 78, 013705.

25 P. Giannozzi, et al., QUANTUM ESPRESSO: A modular and open-source software project for quantum simulations of materials, J. Phys.: Condens. Matter, 2009, 21, 395502.

26 J. P. Perdew, K. Burke and M. Ernzerhof, Generalized gradient approximation made simple, Phys. Rev. Lett., 1996, 77, 3865-3868.

27 A. M. Rappe, K. M. Rabe, E. Kaxiras and J. D. Joannopoulos, Optimized pseudopotentials, Phys. Rev. B: Condens. Matter Mater. Phys., 1990, 41, 1227-1230.

28 J. D. Pack and H. J. Monkhorst, "Special points for Brillouinzone integrations"-a reply, Phys. Rev. B: Condens. Matter Mater. Phys., 1977, 16, 1748-1749.

29 S. Grimme, J. Antony, S. Ehrlich and H. Krieg, A consistent and accurate $\mathrm{ab}$ initio parametrization of density functional dispersion correction (DFT-D) for the 94 elements $\mathrm{H}-\mathrm{Pu}$, J. Chem. Phys., 2010, 132, 154104.

30 M. Methfessel and A. T. Paxton, High-precision sampling for Brillouin-zone integration in metals, Phys. Rev. B: Condens. Matter Mater. Phys., 1989, 40, 3616-3621.

31 G. Henkelman, B. P. Uberuaga and H. Jónsson, A climbing image nudged elastic band method for finding saddle points and minimum energy paths, J. Chem. Phys., 2000, 113, 9901.

32 H. Shin, A. Schwarze, R. Diehl, K. Pussi, A. Colombier, É. Gaudry, J. Ledieu, G. M. McGuirk, L. N. Serkovic Loli, V. Fournée, L. L. Wang, G. Schull and R. Berndt, Structure and dynamics of $\mathrm{C}_{60}$ molecules on $\mathrm{Au}(111)$, Phys. Rev. B: Condens. Matter Mater. Phys., 2014, 89, 245428.

33 C.-T. Tzeng, W.-S. Lo, J.-Y. Yuh, R.-Y. Chu and K.-D. Tsuei, Photoemission, near-edge X-ray absorption spectroscopy, and low-energy electron-diffraction study of $\mathrm{C}_{60}$ on $\mathrm{Au}(111)$ surfaces, Phys. Rev. B: Condens. Matter Mater. Phys., 2000, 61, 2263-2272.

34 E. Abad, J. Ortega and F. Flores, Metal/organic barrier formation for $\mathrm{a}_{60} / \mathrm{Au}$ interface: From the molecular to the monolayer limit, Phys. Status Solidi A, 2012, 209, 636-646.

35 C. J. Villagómez, I. L. Garzón and L. Oliver Paz-Borbón, A first-principles DFT dispersion-corrected $\mathrm{C}_{60} / \mathrm{Au}(111)$ Raman study, Comput. Mater. Sci., 2020, 171, 109208.

36 A. J. Britton, M. Weston, J. B. Taylor, A. Rienzo, L. C. Mayor and J. N. O'Shea, Charge transfer interactions of a Ru(II) dye complex and related ligand molecules adsorbed on $\mathrm{Au}(111)$, J. Chem. Phys., 2010, 133, 094705.

37 J. A. Gardener, G. A. D. Briggs and M. R. Castell, Scanning tunneling microscopy studies of $\mathrm{C}_{60}$ monolayers on $\mathrm{Au}(111)$, Phys. Rev. B: Condens. Matter Mater. Phys., 2009, 80, 235434.

38 K. M. Burson, Y. Wei, W. G. Cullen, M. S. Fuhrer and J. E. Reutt-Robey, Potential steps at $\mathrm{C}_{60}-\mathrm{TiOPc}-\mathrm{Ag}(111)$ interfaces: Ultrahigh-vacuum-noncontact scanning probe metrology, Nano Lett., 2012, 12, 2859-2864. 